\title{
Prominence fine structures in a magnetic equilibrium: Two-dimensional models with multilevel radiative transfer
}

\author{
P. Heinzel ${ }^{1,2}$ and U. Anzer ${ }^{2}$ \\ 1 Astronomical Institute, Academy of Sciences of the Czech Republic, 25165 Ondřejov, Czech Republic \\ 2 Max-Planck-Institut für Astrophysik, Karl-Schwarzschild-Strasse 1, 85740 Garching, Germany
}

Received 10 May 2001 / Accepted 20 June 2001

\begin{abstract}
In this paper we construct theoretical models for vertical prominence threads which are in magnetohydrostatic (MHS) equilibrium. These models are fully two-dimensional (2D) and take the form of vertically infinite threads hanging in a horizontal magnetic field. A typical example of such a $2 \mathrm{D}$ magnetic-dip structure is shown for the case when the central cool parts are surrounded by the prominence-corona transition region (PCTR). We display $2 \mathrm{D}$ variations of the pressure, density and temperature. While the pressure variations follow from the MHS equilibrium, the kinetic temperature was specified empirically. As a next step, we have solved the 2D multilevel non-LTE transfer problem in such thread-like structures, in order to predict the spatial variations of the emergent hydrogen spectrum. It is demonstrated that the hydrogen Lyman lines (treated with partial redistribution) show significant spatial variations of the intensity and that an important difference exists between the line profiles emergent along and across the magnetic field lines. We also discuss how these intensity variations compare to recent SOHO/SUMER prominence observations, namely we show the effects of line-profile averaging over the fine-structure threads which are below the instrumental resolution. Finally we make some suggestions for future modelling and observations.
\end{abstract}

Key words. Sun: prominences - magnetohydrodynamics - radiative transfer - line: profiles

\section{Introduction}

Magnetohydrostatic (MHS) equilibria for quiescent prominences have been first considered by Kippenhahn \& Schlüter $(1957$ - KS). These relatively simple onedimensional (1D) models were proposed to describe the whole prominence in the form of a vertical sheet supported by a dipped magnetic field. Using original KS models, Poland \& Anzer (1971) have attempted to study the global energy balance in quiescent prominences. First multilevel non-LTE models in MHS equilibrium of the KS-type have then been constructed by Heasley \& Mihalas (1976). Similar 1D models but with an empirical prominencecorona transition region (PCTR) were recently published by Anzer \& Heinzel (1999 - AH). All these models consider the variations of the structural parameters only in one dimension, along the magnetic field lines. The radiative transfer is also treated in 1D. While previous MHSmodels were aimed at representing the whole prominence (as 1D vertical slabs), Heinzel \& Anzer (1999) point out that such KS-type models in MHS equilibrium can actually describe fine-structure magnetic dips. However, because of the 1D nature of existing models, one cannot study the variations across the field lines.

Send offprint requests to: P. Heinzel,

e-mail: pheinzel@asu.cas.cz
Prominence observations carried out both from ground and from space show the prominence structure at various angles with respect to the field-line orientation. To interpret such observations properly one has to consider more general MHS models in 2D or 3D. The fact that this aspect can play an important role in interpreting the observed spectra was recently established by a detailed analysis of the SOHO/SUMER spectra of two quiescent prominences (Heinzel et al. 2001). In one case a strong emission without any self-reversal was detected in hydrogen Lyman lines higher than $\mathrm{L} \alpha$, a situation corresponding to MHS models with a PCTR extending along the field lines. The other prominence exhibited a similar brightness in the Lyman spectrum but all lines were strongly reversed. This can be understood in terms of almost isobaric-isothermal models with a steep jump to coronal conditions, a situation which can be met when viewing the structure across the field lines. However, the results presented in that paper were derived from $1 \mathrm{D}$-slab models.

Quiescent prominences exhibit a variety of fine structures, in particular thread-like features which can be vertical, horizontal or at some other inclinations. The vertical threads which are perpendicular to the mean magnetic field could be formed in a way which was proposed by Poland \& Mariska (1988). In their picture a thermal condensation can spread vertically due to the magnetic 
interaction of neighbouring field lines. Such vertical threads will be investigated in the present paper. For horizontal threads, Chiuderi Drago et al. (1992) have proposed a different scenario in which the threads outline horizontal flux tubes. But in this case a very special magnetic configuration would be required with the field being exactly horizontal over very great lengths.

In this paper we generalize the $1 \mathrm{D}$ models of $\mathrm{AH}$ to two dimensions. As a result we obtain MHS equilibria for prominence magnetic dips which take the form of vertically infinite $2 \mathrm{D}$ threads hanging in a horizonal magnetic field. First we present the mathematical formulation of such equilibria and show an example of a typical model structure, i.e. $2 \mathrm{D}$ variations of the gas pressure, density and kinetic temperature (the latter is prescribed semiempirically). As a next step we solve the multilevel nonLTE radiative-transfer problem in such $2 \mathrm{D}$ structures, in order to predict the spatial variations of the emergent hydrogen spectrum. This is achieved by using the same procedure as in Paletou (1995), who solved the multilevel nonLTE problem in isobaric-isothermal 2D prominence slabs. We demonstrate how the synthetic line profiles vary when the prominence thread is seen along and across the field lines and discuss the implications for interpretation of the observed hydrogen spectra, for example those obtained by SOHO/SUMER.

\section{Magnetohydrostatic equilibrium of vertical fine structures}

In this section we model the equilibrium of threads which are uniform in the vertical direction. We shall use the standard Cartesian coordinate system with $x$ normal to the prominence, $y$ along its axis and $z$ pointing vertically upward. Generally the magnetic field in prominences has a strong shear component (e.g. Bommier et al. 1994). But since the presence of a $B_{y}$-component complicates our analysis unnecessarily we shall for simplicity consider here only configurations with $B_{y}=0$ and postpone the modelling of threads with magnetic shear to a later investigation.

Equilibria for 1D slab models with arbitrary temperature variation in the $x$-direction have been constructed in $\mathrm{AH}$. One can generalize these configurations by allowing all quantities to vary also in the $y$-direction. The total pressure will be given by

$p_{\mathrm{T}}=p+\frac{B_{x}^{2}+B_{z}^{2}}{8 \pi}$,

where $p$ is the sum of gas and turbulent pressure. For the case of 1D slabs this total pressure is independent of $x$ and since there is no magnetic tension force in $x$-direction this will lead to equilibrium in $x$-direction. In our new models one also requires pressure equilibrium in the $y$-direction. This then means that $p_{\mathrm{T}}$ has to be uniform in the whole 2D space.
The requirement for vertical equilibrium leads to the equation (see $\mathrm{AH}$ )

$p(m, y)=4 p_{\mathrm{c}}(y) \frac{m}{M(y)}\left[1-\frac{m}{M(y)}\right]+p_{0}$

with the definition

$p_{\mathrm{c}}(y)=\frac{B_{z 1}^{2}(y)}{8 \pi}$

and with $M(y)$ given by

$M(y)=\frac{B_{x}(y) B_{z 1}(y)}{2 \pi g}$.

Here $p_{0}$ is the boundary coronal pressure, $g$ is the gravitational acceleration at the solar surface, $B_{x}(y)$ the horizontal field (constant along a given field-line), $B_{z 1}(y)$ the vertical field at the boundary and $M(y)$ the column mass integrated in the $x$-direction. In this formulation we have introduced the local column-mass coordinate $m$ instead of $x$ which makes the equilibrium equations independent of the assumed temperature profile. For this reason we shall use an $(m, y)$ coordinate system for the equilibrium calculations. Since $p_{\mathrm{T}}$ is constant everywhere we now obtain the relation

$\frac{B_{x}^{2}(y)+B_{z 1}^{2}(y)}{8 \pi}+p_{0}=\frac{B_{x 0}^{2}}{8 \pi}+p_{0}$

or equivalently

$B_{z 1}^{2}(y)=B_{x 0}^{2}-B_{x}^{2}(y)$.

$B_{x 0}$ is the horizontal field which exists in the region between individual threads. Using Eqs. (4) and (6) we can eliminate $B_{x}(y)$ and then obtain

$\frac{B_{z 1}^{2}(y)}{8 \pi}=\frac{1}{2}\left[\frac{B_{x 0}^{2}}{8 \pi}-\sqrt{\left(\frac{B_{x 0}^{2}}{8 \pi}\right)^{2}-\frac{M^{2}(y) g^{2}}{4}}\right]$.

This result now implies that $p(m, y)$ as given by Eqs. (2) and (3) will be completely determined by the choice of the function $M(y)$ and the free parameters $p_{0}$ and $B_{x 0}$. Note that Eq. (7) represents one particular solution valid only when the magnetic dip is shallower than $45^{\circ}$ (i.e. for $B_{x}>B_{z 1}$; we only consider positive values of the field components). Configurations with $B_{x}<B_{z 1}$ will require more complex mass distributions and this will be investigated in another paper.

As we did in our earlier paper $(\mathrm{AH})$, we again specify temperature profiles of the form

$T(m, y)=$

$$
T_{\text {cen }}(y)+\left[T_{\mathrm{tr}}-T_{\mathrm{cen}}(y)\right]\left\{1-4 \frac{m}{M(y)}\left[1-\frac{m}{M(y)}\right]\right\}^{\gamma_{1}}
$$

where the temperature at the boundary $T_{\mathrm{tr}}$ is taken as constant and the exponent $\gamma_{1}$ has to be chosen properly.

As a next step we have to prescribe the thread structure by specifying both $T_{\text {cen }}(y)$ and $M(y)$. We use the following simple functions

$$
\begin{gathered}
T_{\mathrm{cen}}(y)=\left\{\begin{array}{c}
T_{\mathrm{tr}}-\left(T_{\mathrm{tr}}-T_{0}\right)\left(1-\left|\frac{y}{\delta}\right|^{\gamma_{2}}\right) \\
T_{\mathrm{tr}}
\end{array}\right\} \\
\text { for } \quad\left\{\begin{array}{l}
|y| \leq \delta \\
|y|>\delta
\end{array}\right\}
\end{gathered}
$$


and

$$
M(y)=\left\{\begin{array}{c}
M_{0}\left(1-\left|\frac{y}{\delta}\right|^{\gamma_{3}}\right) \\
0
\end{array}\right\} \quad \text { for } \quad\left\{\begin{array}{l}
|y| \leq \delta \\
|y|>\delta
\end{array}\right\} .
$$

Here $M_{0}$ is the maximum column density, $T_{0}$ the minimum central temperature, $2 \delta$ represents the width of each thread and $\gamma^{\prime} s$ are free exponents.

These equations completely describe the thread structure as a function of $m$ and $y$. To obtain the geometrical structure one has to perform a transformation from the mass coordinate $m$ to the spatial coordinate $x$. This requires the following integration

$x=\int_{M(y) / 2}^{m} \frac{\mathrm{d} m^{\prime}}{\rho}$

at each value of the coordinate $y$ ( $\rho$ is the density). The boundaries of the thread are obtained by setting $m=0$ and $m=M(y)$. For the above integration the relation between $p$ and $\rho$ is needed.

The pressure in the thread is given by

$p=p_{\mathrm{g}}+p_{\mathrm{t}}$,

where we include the turbulent pressure $p_{\mathrm{t}}$ (note that in our earlier papers we had neglected the effects of the turbulent pressure on the equilibrium, but we had included the turbulent broadening in the line-profile calculations). A recent study by Heinzel et al. (2001) shows that the Lyman lines observed by SOHO/SUMER indicate the presence of turbulent velocities in the transition region as high as $20 \mathrm{kms}^{-1}$ at temperatures above $10^{4} \mathrm{~K}$. Such velocities can give a sizeable contribution to the pressure term. One can argue that the turbulent velocity is higher in the high temperature regions than in the central cool parts. For simplicity we therefore assume here that the turbulent velocity is a constant fraction of the local sound velocity

$v_{\mathrm{t}}=\epsilon c_{\mathrm{s}}$,

with

$c_{\mathrm{s}}^{2}=\gamma \frac{k T}{\bar{m}}$.

With this assumption we then get

$p=p_{\mathrm{g}}+\frac{1}{2} \rho v_{\mathrm{t}}^{2}=\rho k T \frac{1+0.5 \gamma \epsilon^{2}}{\bar{m}}$.

Here the mean molecular mass $\bar{m}$ is given by

$\bar{m}=\frac{1+4 \alpha}{1+\alpha+i} m_{\mathrm{H}}$,

where $\alpha$ is the ratio of helium to hydrogen particle densities, $i$ the local ionization degree of hydrogen (here we do not consider the helium ionization), $m_{\mathrm{H}}$ the mass of the hydrogen atom and $\gamma$ is the ratio of specific heats. For our special case of a constant ratio between $v_{\mathrm{t}}$ and $c_{\mathrm{S}}$ one can therefore use the normal ideal gas law if one replaces the mass $m_{\mathrm{H}}$ by the reduced mass given by $m_{\mathrm{H}} /\left(1+0.5 \gamma \epsilon^{2}\right)$.
With $\gamma=5 / 3, \alpha=0.1$ and $i=0.3$ one gets $c_{\mathrm{s}}=$ $11.8 \mathrm{~km} \mathrm{~s}^{-1}$ at $T=10^{4} \mathrm{~K}$.

Finally, note that Low (1982) has also modelled vertical threads in prominences. He has taken a special class of analytical equilibrium solutions for which all physical variables are separable, i.e. can be represented by functions of the form $f_{1}(x) f_{2}(y)$. This assumption restricts the possible models considerably. His models have also the property that along each individual field line the temperature is constant. Because of all these limitations we cannot use these models for our investigation of prominence fine structure.

\section{2D models of magnetic dips}

As an example of the magnetically confined thread we consider here a structure which is $10^{3} \mathrm{~km}$ thick in $y$-direction and more extended in the $x$-direction. While the thickness in the $y$-direction is arbitrary, the extension along the magnetic field lines results from the given model for the pressure equilibrium. We thus model a dip-like structure elongated along the field lines and infinitely extended in the vertical direction (2D models). This can be identified with vertical fine-structure threads. The model we take for the pressure equilibrium belongs to the class of models discussed in $\mathrm{AH}$, but here we have assumed a somewhat increased coronal pressure $p_{0}$. The model parameters are:

$M_{0}=10^{-4} \mathrm{~g} \mathrm{~cm} \mathrm{~cm}^{-2}, \quad p_{0}=0.03 \mathrm{dyn}_{\mathrm{cm}^{-2}}$, $B_{x}(0)=4.5$ Gauss, $T_{0}=8000 \mathrm{~K}, T_{\mathrm{tr}}=5 \times 10^{4} \mathrm{~K}$, $2 \delta=10^{3} \mathrm{~km}, \gamma_{1}=5, \gamma_{2}=60, \gamma_{3}=2, \alpha=0.1$ and $\epsilon=0.5$.

Note that here we used as the input parameter $B_{x}(0)$ (field in the middle of the thread) instead of $B_{x 0}$ (field between threads). This is because the former one is a measurable quantity more or less known for quiescent prominences (Bommier et al. 1994). $B_{x 0}$ and $B_{x}(0)$ are simply related through Eqs. (4) and (6) with $M_{0}$ given as the input parameter. $B_{z 1}(0)$ is then equal to 3.83 Gauss (i.e. lower than $B_{x}(0)$ as required).

With this set of input parameters we have computed the model in pressure equilibrium, with empirical temperature structure according to Eq. (8). The results are shown in Figs. 1-3, where we display 2D variations of temperature $T$, pressure $p$ and gas density $\rho$, respectively. Note that $p$ consists of the gas pressure and the turbulent pressure - see Eq. (15). The density structure shown in Fig. 3 is computed from a simple estimate of the ionization-degree variations with $T$

$i=1-\left(1-i_{\mathrm{c}}\right)\left(\frac{T_{\mathrm{tr}}-T}{T_{\mathrm{tr}}-T_{\text {cen }}}\right)^{2}$,

where we have taken $i_{\mathrm{c}}=0.3$ at the dip center as suggested from the results of AH. Then the $x$-scale, consistent with this distribution, is held fixed for 2D non-LTE computations, while the ionization degree and the gas density 


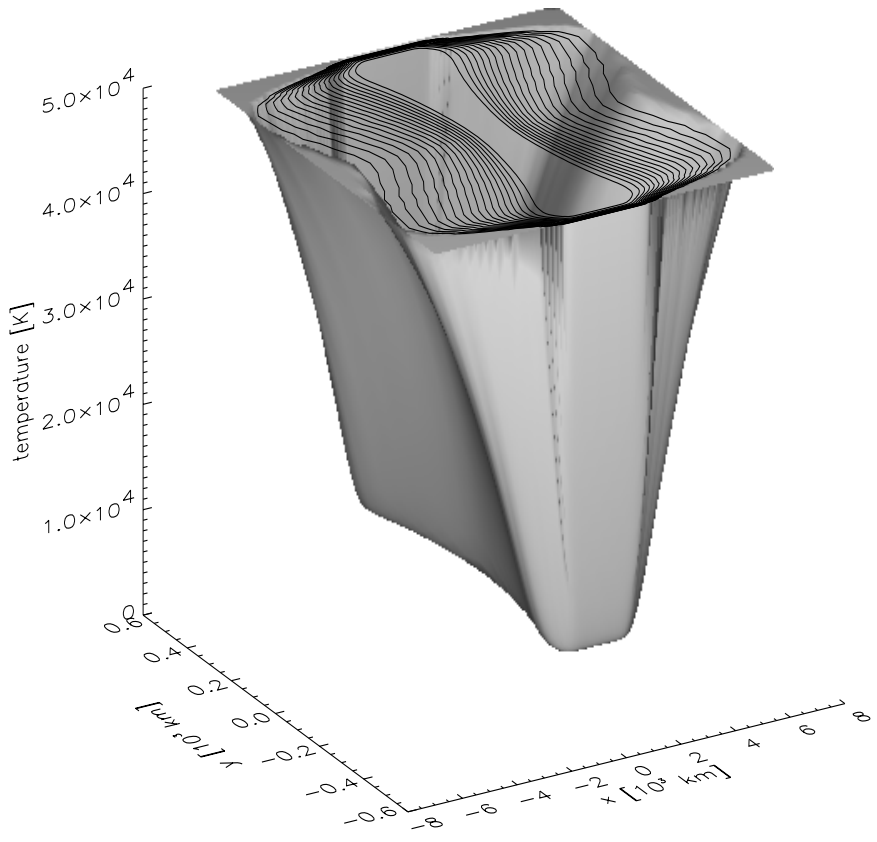

Fig. 1. Temperature structure of a 2D magnetic dip. Note that the plot is considerably compressed in the $x$-direction.

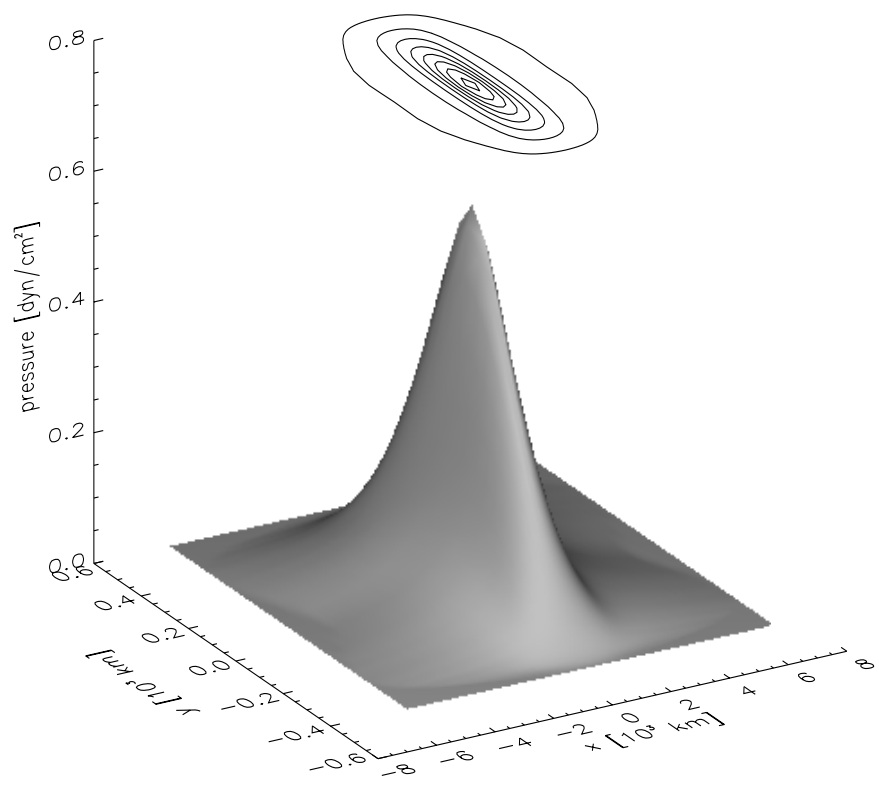

Fig. 2. Pressure structure of a 2D magnetic dip.

are properly iterated. More consistent calculations should perform a re-scaling of the $x$-coordinate according to the current structure of the ionization degree, an effect which we neglect in these first exploratory models.

The temperature structure along the field lines is similar to those considered in $\mathrm{AH}$ for $1 \mathrm{D}$ models. However, across the field lines we consider a very steep temperature rise caused by magnetically shielded transition from cool to hot coronal plasmas. This is clearly demonstrated in Fig. 1 - see variation in $y$-direction. Such a temperature structure was recently deduced by

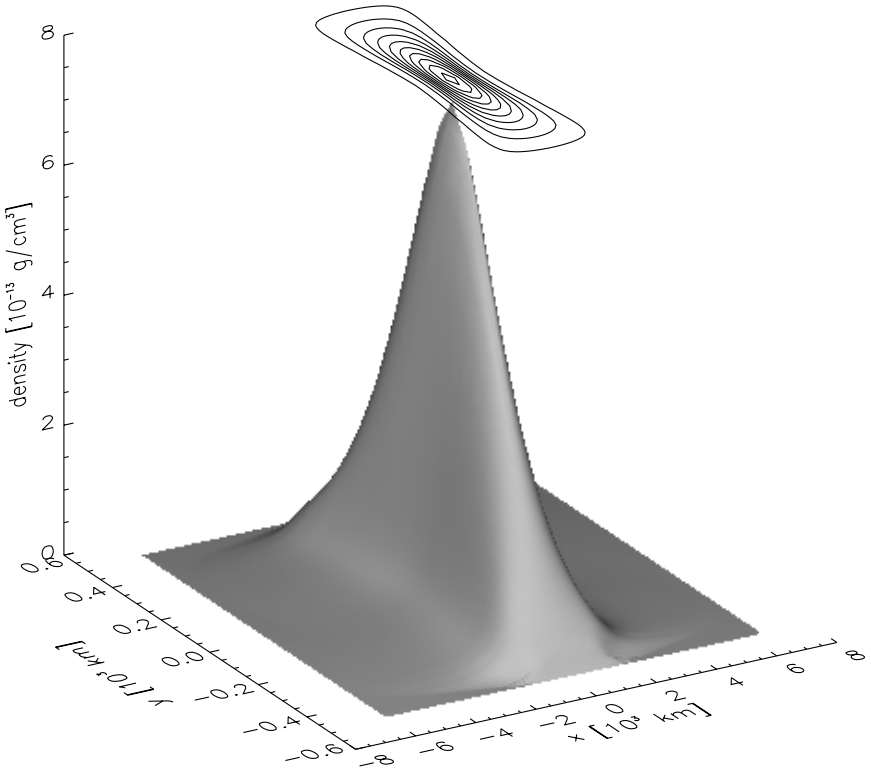

Fig. 3. Density structure of a 2D magnetic dip.

Heinzel et al. (2001) from SOHO/SUMER prominence observations of the Lyman spectrum.

In this particular model, the pressure isocontours (Fig. 2) show approximately an oval shape, while the gas density is characterized by isocontours somewhat squeezed in the $x$-direction (a similar shape can be seen also in the temperature plot). We call this a "horn-like" shape; it can be much more pronounced for other models of interest and we will study this behaviour in future papers. Our present selection of the model with very marginal hornlike structure was intentional because pronounced horns largely complicate the proper selection of a 2D Cartesian grid on which the transfer problem is solved (see below).

Note that while the pressure outside the dip boundary is almost constant (the scale-height is large), the temperature should further increase and the density decrease. But here we have fixed the latter two to the boundary values which simplifies the modelling and has not significant effects on our results.

Finally, we plot in Fig. 4 the magnetic surface of the dip, computed by integration of the field-line equation

$\frac{\mathrm{d} z}{\mathrm{~d} x}=\frac{B_{z}}{B_{x}}$,

with the condition that the height $z_{0}$ at $p=p_{0}$ is the same for all field lines. In our 2D model, the magnetic dip represents a local (fine-structure) feature modelled only to its boundaries. Then the central field line has the inclination at the surface equal to $38.7^{\circ}$ and the outermost field line is horizontal with $B_{x}=B_{x 0}$ and $B_{z}=0$. Note that this inclination is directly related to the plasma $\beta$-parameter at the dip center (Heinzel \& Anzer 1999).

For our models the magnetic field at the thread boundary can be matched to the following force-free field (with currents) in the surrounding corona: the field strength is 


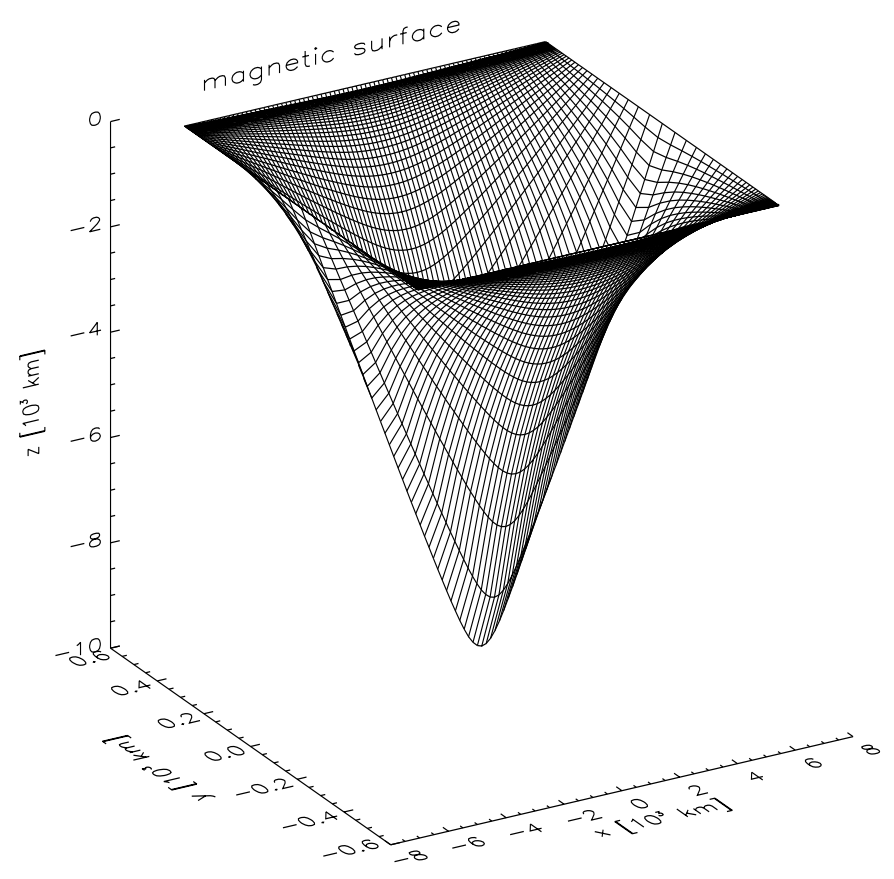

Fig. 4. Magnetic-surface structure of a 2D magnetic dip. Here the wire-lines lying in the vertical $x-z$ planes represent the magnetic field lines.

constant everywhere $\left(B=B_{x 0}\right)$ and the orientation rotates around the $y$-direction in the appropriate way. But this will only be true in the neighbourhood of the prominence. At large distances all field lines will have to bend to connect to the solar surface and thereby form a magnetic arcade.

\section{2D multilevel radiative transfer}

Our two-dimensional dips described in the preceding sections form threads with uniform properties along the vertical direction. To solve the radiative-transfer problem, we can thus treat them as $2 \mathrm{D}$ structures infinite in vertical extension ( $z$-direction) and having rather complicated variations in the perpendicular $x-y$ plane. We assume that each thread is irradiated by the incident solar radiation and these threads do not interact radiatively.

An efficient method for solving 2D transfer problems in prominence-like structures was developed by Auer \& Paletou (1994). The method is based on the Accelerated Lambda Iteration (ALI) technique and uses the short characteristics (SC) to obtain the formal solution along individual rays. The hydrogen multilevel problem was addressed for 2D structures by Paletou (1995, 1996), who also suggested a simple iterative procedure to treat partially-coherent scattering (so-called partial redistribution - PRD) in resonance lines. The non-LTE statisticalequilibrium equations are preconditioned according to Rybicki \& Hummer (1991, 1992) (so-called MALI technique) and then linearized with respect to particle number densities to compute the hydrogen ionization balance (Heinzel 1995; Paletou 1995). This 2D multilevel method was successfully applied by Paletou (1995) to isobaric-isothermal prominences having infinite horizontal extension in one direction, i.e. the whole prominence was treated as a $2 \mathrm{D}$ slab of a finite thickness and vertical extension.

Although our geometry of vertical threads with $2 \mathrm{D}$ cross-section is different from the models of Paletou, the same SC technique can be used to solve the $2 \mathrm{D}$ transfer problems. We thus fully reproduced the methods described in Auer \& Paletou (1994) and in Paletou (1995) and developed an appropriate 2D solver which was then integrated into our multilevel non-LTE prominence code (Heinzel 1995, AH). Extensive tests have been made, particularly by comparing $2 \mathrm{D}$ solutions for vertical threads extended horizontally in one dimension with solutions obtained for vertical 1D slabs. More specifically, for an isothermal and isobaric structure, which is very elongated in the $y$-direction, we computed the $2 \mathrm{D}$ model and then we compared the variations of the source functions along the $x$-direction at the middle of the structure with the 1D model which corresponds to an infinite extension in $y$-direction. It gave the same results, although in $1 \mathrm{D}$ we have used the LC (long-characteristics) method with the Feautrier formal solver instead of SC. The number of rays per octant was chosen to be 12 and we used the abscissae and weights from Carlson (1963).

However, in contrast to Paletou (1995, 1996), our $2 \mathrm{D}$ structures are not isobaric and have significant temperature variations inside the PCTR. This requires a careful treatment of the $2 \mathrm{D}$ grid defined on the rectangular boxes shown in Figs. 1-3. First, the structures generally exhibit "horn-like" features and second, we have to consider large spatial variations of all thermodynamic parameters. In the model considered in this paper, the variations along the $x$-axis are rather smooth with decreasing density and increasing ionization degree towards the boundary - this suggests an equidistant grid for the $x$-axis. On the other hand, temperature variations along the $y$-axis are so steep (with $\gamma_{2}=60$ which mimics a sudden jump in temperature across the field lines) that we have to use a logarithmic spacing to resolve the boundary layers. In a general case with pronounced horns, a special kind of "mesh-refinement technique" has to be considered.

For these first exploratory calculations we use an isotropic mean incident solar radiation, which has been calculated by integrating the observed disk intensities taking into account their center-to-limb variations (for details see Heinzel 1995). The adopted height above the solar surface was $10^{4} \mathrm{~km}$. These incident radiation fields are the same as for $1 \mathrm{D}$ vertical slabs, in contrast to irradiation of horizontal 2D structures discussed by Paletou (1996). The hydrogen atomic data and other computational details are also described in Heinzel (1995).

\section{Output radiation from dips}

For the thread model shown in Sect. 3 we have performed $2 \mathrm{D}$ non-LTE transfer computations using the 


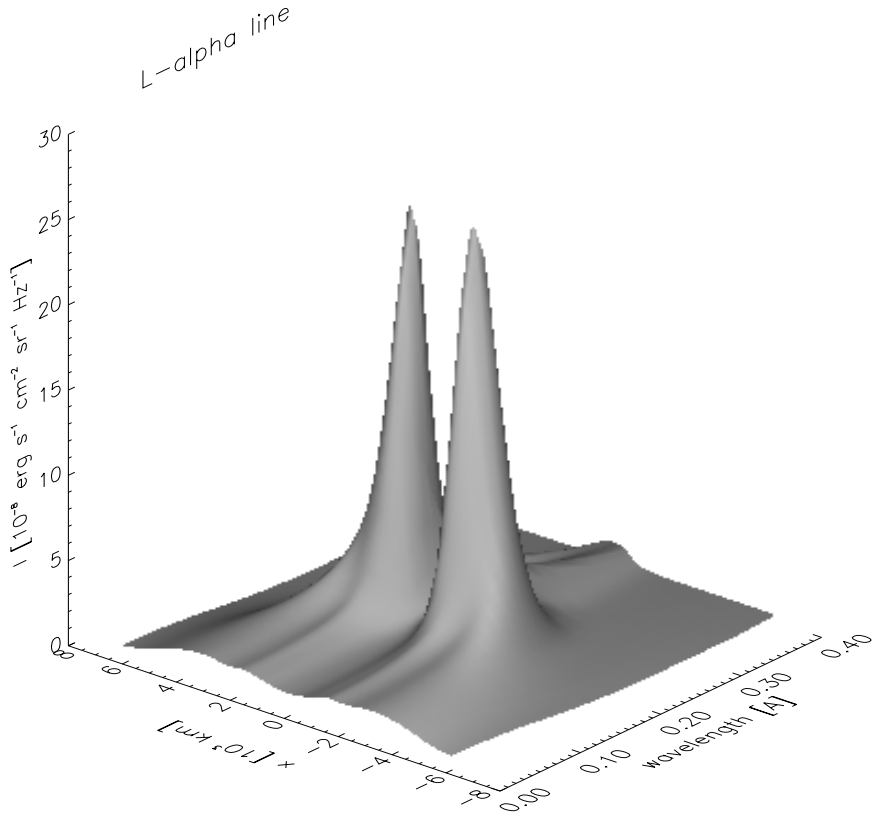

Fig. 5. L $\alpha$ intensity variations seen along the $x$-axis.

MALI method described in the previous section. We have used a 5-level plus continuum hydrogen model atom and the partial redistribution for $\mathrm{L} \alpha$ and $\mathrm{L} \beta$ lines (higher Lyman lines are less sensitive to PRD effects). The convergence properties are very good, the method of Auer \& Paletou (1994) has been proven to be robust enough and stable even for such strongly varying conditions as considered in our new models, provided that we use a linear interpolation between the grid points for all quantities other than the source function. For the source function we use the parabolic fit as in Auer \& Paletou (1994). Parabolic interpolation for other quantities will certainly lead to somewhat better accuracy, but may also cause unexpected instabilities owing to the strong gradients considered in our models. We did not investigate this so far, but our tests with the independent 1D calculations gave satisfactory results. The Cartesian grid consisted of $83 \times 83$ points and we have used 12 rays/octant to describe the angular variations of the radiation field. We have taken advantage of symmetry properties of the dip, i.e. the fact that the dip cross-section is symmetrical in the four quadrants.

From the converged model we finally evaluate the line radiation emerging along all grid lines. To visualize it, we plot one half of each (symmetrical) profile emergent either along the $x$-directions (the axis in the plot is then $y$ ) or along the $y$-directions (the axis in the plot is $x$ ). In the former case we look along the field lines, in the latter across them. In the following text we say "intensity variations along $x$ and $y$ axis" for radiations emergent along $y$ and $x$-directions (grid lines), respectively. The corresponding plots in 3D graphics are shown as shaded surfaces of line intensities in Figs. 5-10, for three lines: $\mathrm{L} \alpha, \mathrm{L} \beta$ and $\mathrm{H} \alpha$.

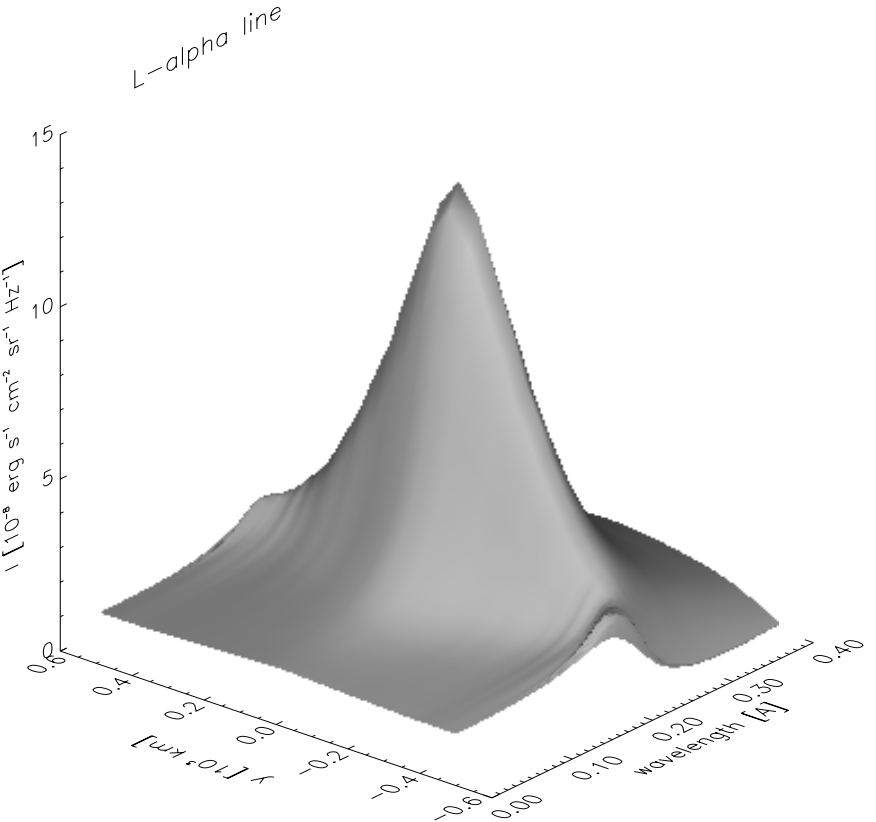

Fig. 6. $\mathrm{L} \alpha$ intensity variations seen along the $y$-axis.

From these 3D plots one can see strong intensity variations along both axes in all three lines. These variations are caused both by $2 \mathrm{D}$ transfer effects (even when the irradiation of the dip is isotropic), as well as by strong variations of the temperature and density. The behaviour of both Lyman lines is similar and reflects the fact that their cores are formed inside the PCTR. $\mathrm{H} \alpha$ is quite different, formed in central cool parts of the dip.

The basic feature of Lyman lines is that when the dip is viewed along the field lines, the $y$-variations of the profiles show a dominant peak at the dip center, while looking across the field lines we see two sharp maxima. This is due to temperature and density structure of the $2 \mathrm{D}$ dip which is mapped into the variations of the line source functions. In the first case the temperature is practically constant and only at boundaries it jumps to PCTR values. Then the intensity variations along $y$-axis are mainly due to column density decrease towards boundaries (see Eq. (10)). Only close to boundaries small intensity enhancements are visible which correspond to the PCTR region. The line profile emergent at $y=0 \mathrm{~km}$ is similar in nature to those computed from 1D pressure-equilibria models $(\mathrm{AH})$. A quite different situation is found when looking across the field lines. Two strong peaks correspond to regions of enhanced temperature which are symmetrically placed with respect to the dip center at $x=0 \mathrm{~km}$. At positions of these peaks, the transfer along the $y$-direction (y-grid lines) roughly corresponds to that in an almost isothermal model. Strong line reversals are typical for isothermal models at higher temperatures as already shown for 1D models by Gouttebroze et al. (1993).

The $\mathrm{H} \alpha$ line has no reversal in this model but is saturated in the densest central parts. The difference between $x$ and $y$-variations corresponds again to the 


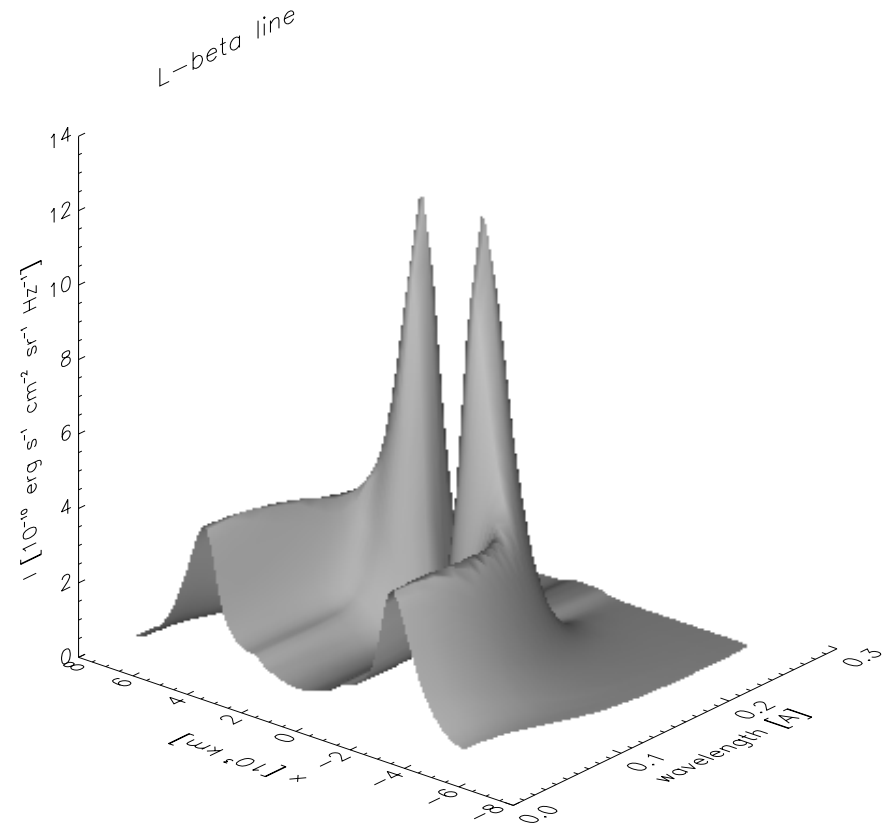

Fig. 7. $\mathrm{L} \beta$ intensity variations seen along the $x$-axis.

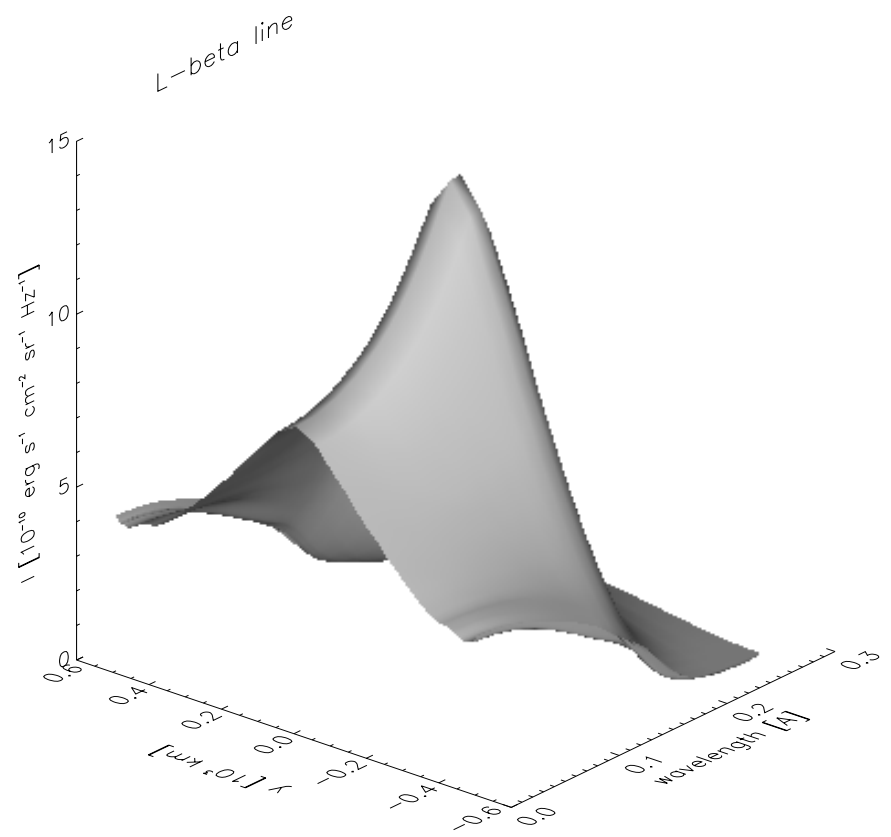

Fig. 8. $\mathrm{L} \beta$ intensity variations seen along the $y$-axis.

temperature and density structure of the dip. $\mathrm{H} \alpha$ is formed in the central cool parts of the dip which is clearly seen by comparing Fig. 1 with Figs. 9 and 10.

\section{Discussion and conclusions}

Our 2D models of magnetic dips represent an important step towards understanding the fine structure of quiescent prominences. $2 \mathrm{D}$ calculations show that the profiles of the different hydrogen lines can vary considerably across the thread and also that they depend on the viewing angle

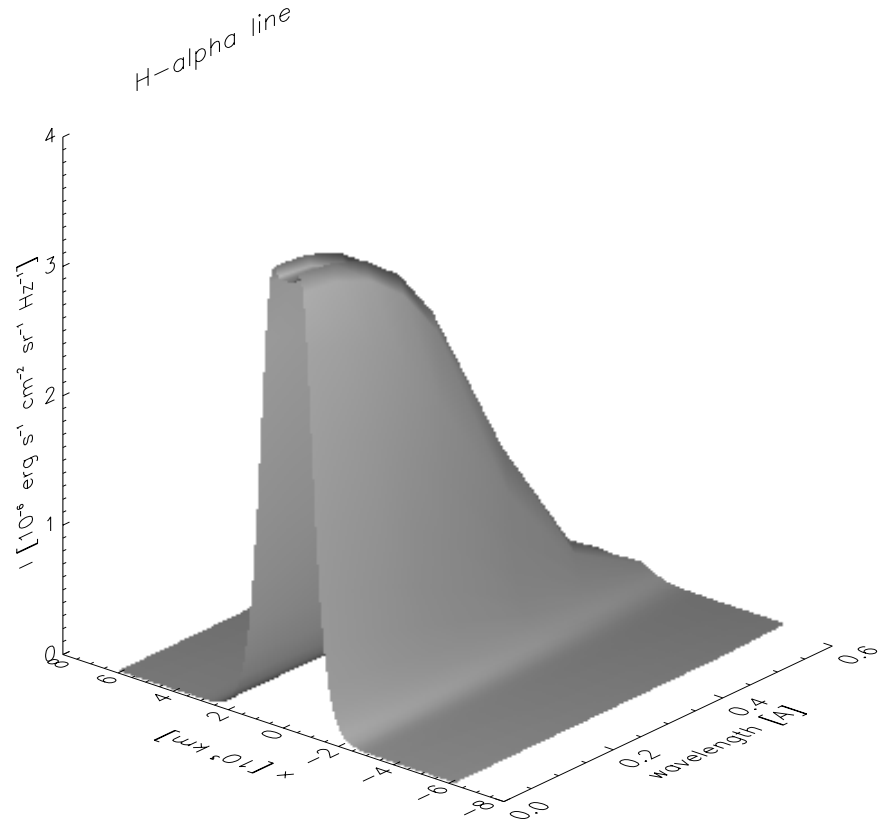

Fig. 9. $\mathrm{H} \alpha$ intensity variations seen along the $x$-axis.

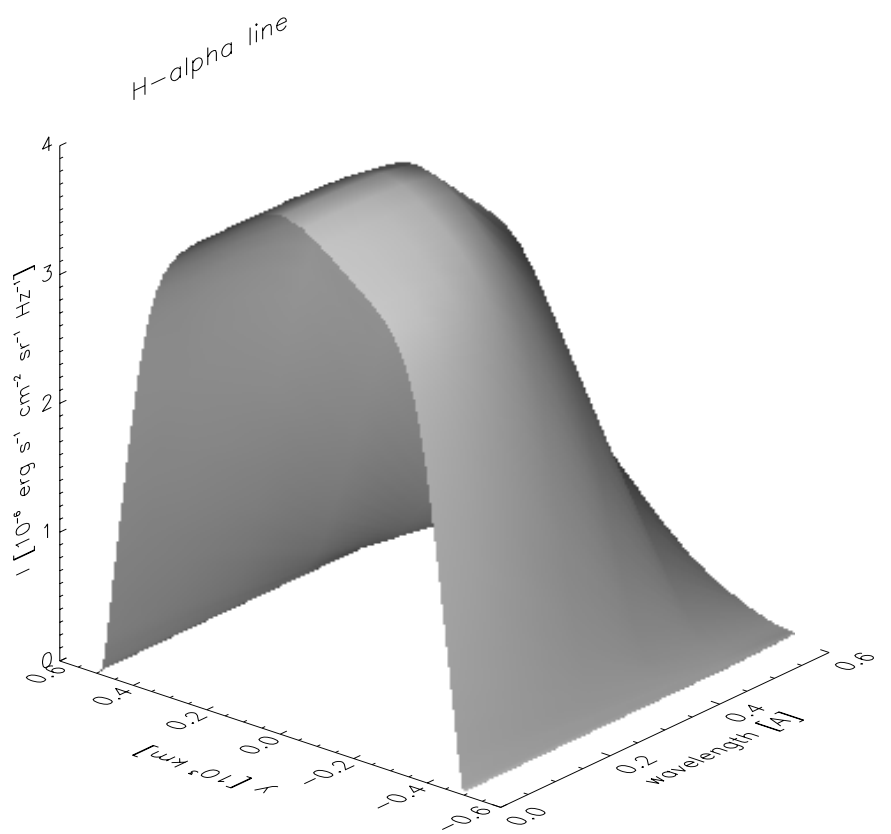

Fig. 10. $\mathrm{H} \alpha$ intensity variations seen along the $y$-axis.

with respect to the magnetic-field orientation. A thickness of the order of $10^{3} \mathrm{~km}$ might be of particular interest for our investigations. When we observe the Lyman lines in prominences by the SOHO/SUMER UV spectrograph, the spatial resolution along the slit is around 1.5 arcsec, which means about $10^{3} \mathrm{~km}$. Therefore, the line profiles are integrated through the structure and this has to be taken into account when interpreting the observed spectra. The effect is very important as one can expect looking at our $2 \mathrm{D}$ variations of the radiative output. In Fig. 11 we show the $\mathrm{L} \beta$ line profile variations in the $y$-direction 


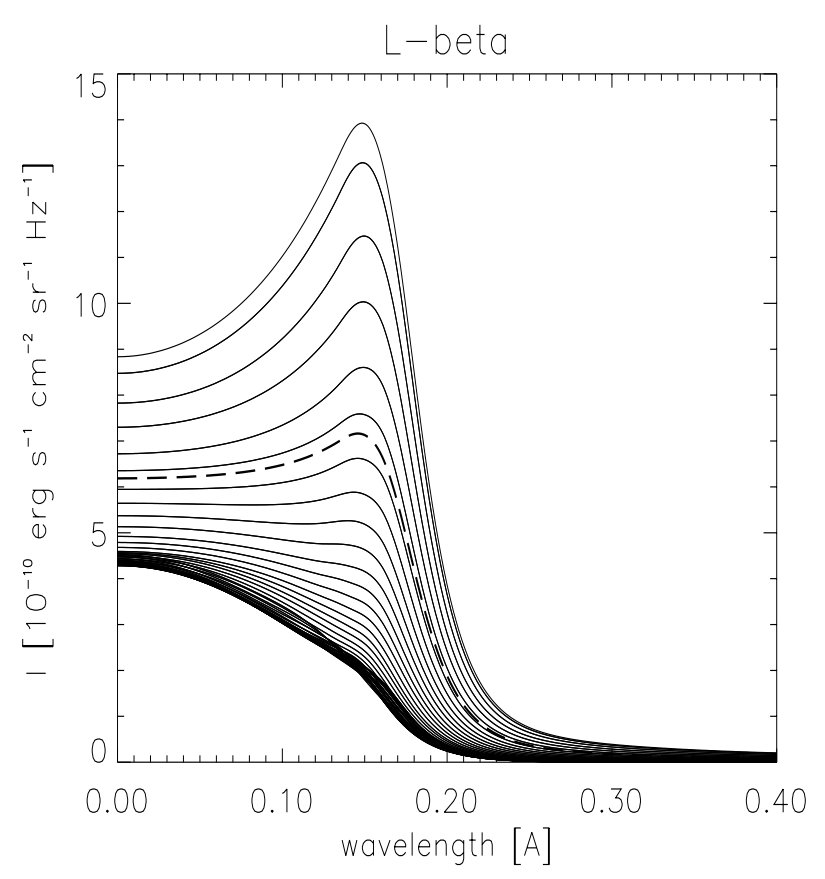

Fig. 11. $\mathrm{L} \beta$ intensity variations seen along the $y$-axis, from the dip center to its boundary: full lines represent all profile variations, dashed line is their mean. Steps along the $y$-axis are logarithmic.

(from dip center to edge) and the averaged profile which results from the integration along the dip thickness $2 \delta$. This has to be compared with SUMER observations of Heinzel et al. (2001) assuming that the vertical threads are more or less perpendicular to the slit orientation (we use here the results for $\mathrm{L} \beta$ because the $\mathrm{L} \alpha$ observed profiles are strongly affected by instrumental effects). When this rather elongated dip is viewed along the $x$-axis (i.e. perpendicularly to field lines), one can still see significant variations - we show them in Fig. 12, together with an averaged profile (over about $1370 \mathrm{~km}$ which is the distance of the sharp peak from the dip center in Fig. 7). Both averaged profiles in Figs. 11 and 12 compare well to the two types of profiles observed by SUMER (Heinzel et al. 2001), although they are somewhat narrower (which is due to lower $v_{\mathrm{t}}$ used in this study and because we neglect the broadening caused by line-intensity integration through several threads). Note that actual spatial resolution with which the Lyman-line profiles used in Heinzel et al. (2001) have been obtained is even lower due to an averaging of the data over several spatial pixels along the slit, in order to improve the photon statistics.

The lowest intensities in Fig. 11 correspond to narrow outermost regions in the $y$-direction where the temperature gradient is very steep. Although these intensities are relatively high (namely at the line center), they contribute only marginally to the mean profile. But here we have to mention two points. First, a significant contribution to these intensities comes from regions outside the dip, i.e. regions where $T=T_{\mathrm{tr}}=$ const. In future models this region should be modelled with a more realistic

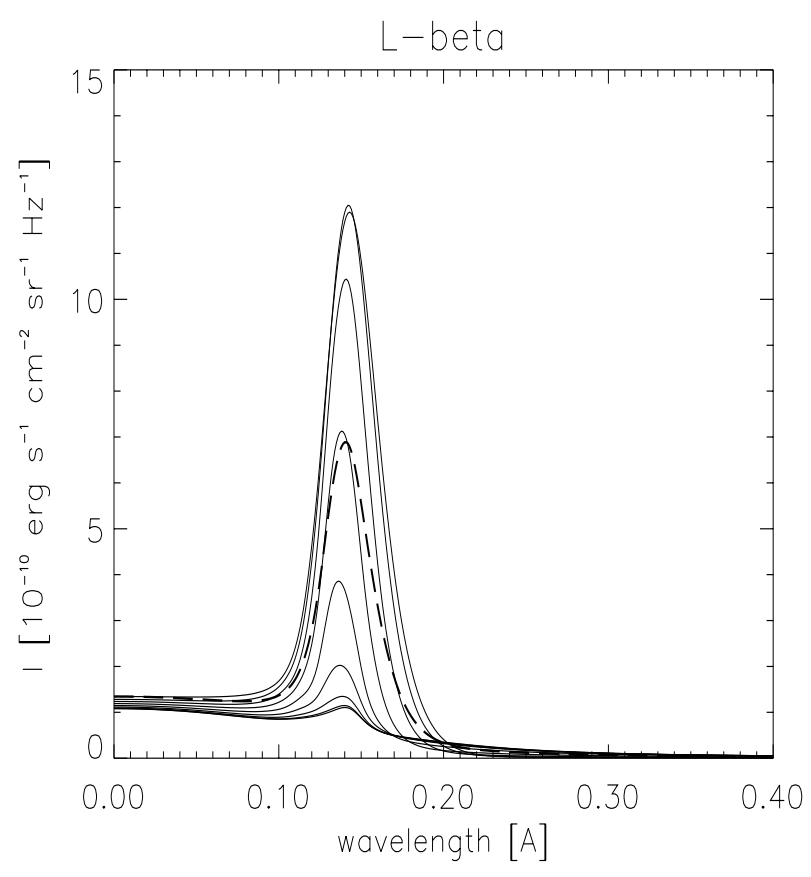

Fig. 12. $\mathrm{L} \beta$ intensity variations seen along the $x$-axis: full profiles correspond to the region between the dip center and about $1370 \mathrm{~km}$ towards the surface, dashed line is their mean. Steps along $x$-axis are equidistant.

temperature gradient. Second, even when a more realistic model of the outer PCTR will be considered, it seems that these high-temperature regions still contribute to Lymanline intensities. This has to be investigated in detail since it can influence the synthetic spectra and also will modify the irradiation of cool parts (with possible contribution to their heating).

The fact that our relatively simple 2D thread models can reproduce the observed line features presented in Heinzel et al. (2001) so well seems very promising. But in order to obtain a more consistent picture, further observations will be needed. Of particular interest will be a statistical study of the dependence of the line profiles on the orientation of the magnetic field with respect to the line of sight. In addition, one should try to obtain spatially-resolved line profiles which could be compared with our calculated profile variations.

Acknowledgements. P. H. acknowledges the support from MPA Garching and from the grant No. A3003902 of the Academy of Sciences of the Czech Republic. U. A. thanks the Ondřejov Observatory for financial support. The authors are indebted to Drs. P. Gouttebroze and F. Meyer for their valuable comments.

\section{References}

Anzer, U., \& Heinzel, P. 1999, A\&A, 349, 974 (AH)

Auer, L. H., \& Paletou, F. 1994, A\&A, 285, 675

Bommier, V., Landi degl'Innocenti, E., Leroy, J.-L., \& Sahal-Bréchot, S. 1994, Solar Phys., 154, 231

Carlson, B. G. 1963, in Methods in Computational Physics, ed. B. Alder, S. Fernbach, \& M. Rotenberg, Statistical Physics, vol. 1 (Academic Press, New York and London), 1 
Chiuderi Drago, F., Engvold, O., \& Jensen, E. 1992, Solar Phys., 139, 47

Gouttebroze, P., Heinzel, P., \& Vial, J.-C. 1993, A\&AS, 99, 513

Heasley, J. N., \& Mihalas, D. 1976, ApJ, 205, 273

Heinzel, P. 1995, A\&A, 299, 563

Heinzel, P., \& Anzer, U. 1999, Solar Phys., 184, 103

Heinzel, P., Schmieder, B., Vial, J.-C., \& Kotrč, P. 2001, A\&A, 370,281

Kippenhahn, R., \& Schlüter, A. 1957, Z. Astrophys., 43, $36(\mathrm{KS})$
Low, B. C. 1982, Solar Phys., 75, 119

Paletou, F. 1995, A\&A, 302, 587

Paletou, F. 1996, A\&A, 311, 708

Poland, A. I., \& Anzer, U. 1971, Solar Phys., 19, 401

Poland, A. I., \& Mariska, J. T. 1988, in Dynamics and Structure of Solar Prominences, Proc. of a Workshop held at Palma de Mallorca, ed. J. L. Ballester, \& E. R. Priest (Université des Iles Baléares), 133

Rybicki, G. B., \& Hummer, D. G. 1991, A\&A, 245, 171

Rybicki, G. B., \& Hummer, D. G. 1992, A\&A, 262, 209 